\title{
Bacterial neuraminidase facilitates mucosal infection by participating in biofilm production
}

\author{
Grace Soong, ${ }^{1}$ Amanda Muir, ${ }^{1}$ Marisa I. Gomez, ${ }^{1}$ Jonathan Waks, ${ }^{1}$ Bharat Reddy, ${ }^{1}$ \\ Paul Planet, ${ }^{1}$ Pradeep K. Singh,, ${ }^{2}$ Yukihiro Kanetko, ${ }^{2}$ Matthew C. Wolfgang, ${ }^{3}$ \\ Yu-Shan Hsiao, ${ }^{4}$ Liang Tong, ${ }^{4}$ and Alice Prince ${ }^{1}$
}

\author{
1Departments of Pediatrics and Pharmacology, College of Physicians and Surgeons, Columbia University, New York, New York, USA. \\ 2Department of Microbiology, University of Washington School of Medicine, Seattle, Washington, USA. 'Department of Microbiology and Immunology, \\ University of North Carolina at Chapel Hill, Cystic Fibrosis Pulmonary Research and Treatment Center, Chapel Hill, North Carolina, USA. \\ ${ }^{4}$ Department of Biological Sciences, Columbia University, New York, New York, USA.
}

\begin{abstract}
Many respiratory pathogens, including Hemophilus influenzae, Streptococcus pneumoniae, and Pseudomonas aeruginosa, express neuraminidases that can cleave $\alpha 2,3$-linked sialic acids from glycoconjugates. As mucosal surfaces are heavily sialylated, neuraminidases have been thought to modify epithelial cells by exposing potential bacterial receptors. However, in contrast to neuraminidase produced by the influenza virus, a role for bacterial neuraminidase in pathogenesis has not yet been clearly established. We constructed a mutant of $P$. aeruginosa PAO1 by deleting the PA2794 neuraminidase locus $(\Delta 2794)$ and tested its virulence and immunostimulatory capabilities in a mouse model of infection. Although fully virulent when introduced i.p., the $\Delta 2794$ mutant was unable to establish respiratory infection by i.n. inoculation. The inability to colonize the respiratory tract correlated with diminished production of biofilm, as assessed by scanning electron microscopy and in vitro assays. The importance of neuraminidase in biofilm production was further demonstrated by showing that viral neuraminidase inhibitors in clinical use blocked $P$. aeruginosa biofilm production in vitro as well. The $P$. aeruginosa neuraminidase has a key role in the initial stages of pulmonary infection by targeting bacterial glycoconjugates and contributing to the formation of biofilm. Inhibiting bacterial neuraminidases could provide a novel mechanism to prevent bacterial pneumonia.
\end{abstract}

\section{Introduction}

Neuraminidases (sialidases) are produced by a wide variety of mucosal pathogens, ranging from Streptococcus pneumoniae in the airway to Vibrio cholerae in the gut (1). While the central role of viral neuraminidase in pathogenesis of influenza is well established (2) and provides a target for both vaccines and chemotherapy, the contribution of bacterial neuraminidase to the pathogenesis of infection is not as clearly defined. Neuraminidase-producing species such as Hemophilus (3), S. pneumoniae (4, 5), and Psendomonas aeruginosa (6) share a common ecological niche, colonizing the heavily sialylated secretions and surfaces of the upper respiratory tract. Although each can bind to asialylated glycolipids exposed by neuraminidase activity (7), they differ substantially in their ability to either metabolize (8) or incorporate sialic acid into surface structures (9). Thus it is likely that bacterial neuraminidases interact with both microbial and eukaryotic glycoconjugates (1).

$P$. aeruginosa is a major opportunistic pathogen, an important cause of nosocomial pneumonia as well as the chief cause of lung infection in cystic fibrosis (CF). Over 2 decades ago, neuraminidase production in isolates of $P$. aeruginosa from $C F$ patients was described and suggested to contribute to pulmonary infection (10). In vitro studies documented that many pulmonary pathogens including $P$. aeruginosa bind to the GalNAc $\beta 1,4 \mathrm{Gal}$ moiety exposed on asialylated glycolipids (7), suggesting that the abil-

Nonstandard abbreviations used: asialoGM1, Gal $\beta 1,2$ GalNAc $\beta 1,4 \mathrm{Gal} \beta 1,4 \mathrm{Gl} \beta \beta 1$, $1 \mathrm{Cer}$, asialoganglioside gangliotetraosylceramide; CF, cystic fibrosis; LB, Luria broth; OMP, outer membrane protein; PMN, polymorphonuclear leukocyte.

Conflict of interest: The authors have declared that no conflict of interest exists. Citation for this article: J. Clin. Invest. 116:2297-2305 (2006). doi:10.1172/JCI27920. ity to desialylate mucosal surfaces could contribute to bacterial colonization of the airways. The $P$. aeruginosa neuraminidase was cloned and characterized. It was shown to be osmoregulated, thought to be consistent with expression in the milieu of the CF lung (6) and capable of exposing the receptor asialoganglioside gangliotetraosylceramide (Gal $\beta 1,2 \mathrm{GalNAc} \beta 1,4 \mathrm{Gal} \beta 1,4 \mathrm{Glc}$ $\beta 1,1 \mathrm{Cer}$ ) (asialoGM1) on the surface of CF airway cells in vitro, implying a role in pathogenesis (11). However, data confirming $P$. aeruginosa adherence to the airway surface in CF patients has been lacking (12). The current consensus suggests that organisms are predominantly entrapped in dehydrated secretions of the lung and - by shedding proinflammatory products - activate airway inflammation (13), a model that does not require direct attachment of organisms to the epithelial surface. Nonetheless, analyses of $P$. aeruginosa gene expression in CF patients document that the PA2794 neuraminidase locus is one of the most highly expressed genes in this patient population in vivo (14). Unlike other respiratory pathogens, $P$. aeruginosa cannot use sialic acid as a carbon source, nor does it contain sialic acid as a component of its LPS (15). Thus it seemed likely that there was some additional function for the enzyme relevant to the pathogenesis of respiratory tract infection. To better define the importance of bacterial neuraminidases, we constructed a $P$. aeruginosa mutant lacking the PA2794 nanA locus and analyzed it in a mouse model of infection. Our studies showed that the $P$. aeruginosa neuraminidase is involved in biofilm formation contributing to initial colonization of the airway. Furthermore, we demonstrated that this activity can be blocked by viral neuraminidase inhibitors in clinical use indicating a novel therapeutic target for preventing bacterial pneumonia. 


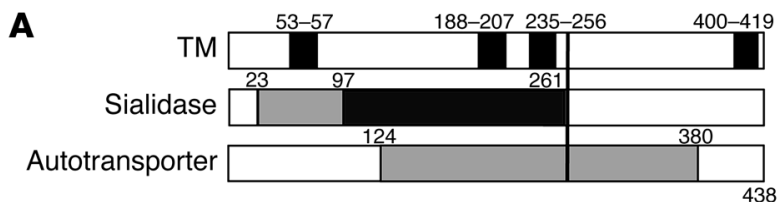

MNTYFDIPHR

RATEHVGGSD

AGGTMPSGRI

ARGGADYNFA

SDGGETWGEG

SGAGGALROF

LSYIYSEGGT

GWTERVPVYS

SGAYQFEVYI

RVVVEFARSS

NIRLGTGAAV
YDHFGQMDIL

GIWSAPTIVA

EVKVYVSDDS

YVIPLYAATG

NETSYLPVGD

DQGNVTAQNG

TTHFCYYRTI

VVLGGRRILG

FSVSSNSLYT

SYFNDGGHKG

NSATTRFATG
SDGSLYLIYR

QAGGQDFRDV

GVTWVHKFTL

VNYELKWLES

GVILAVARVG

DSTDILVAPS

LLAKAVAGSS

NLFRETSSTT

LSHGLQRSPR

SGAQVEVGSL

YYRVRAWIZ

ASP boxes - Ser/Thr - X-Asp-X-Gly-X-Thr-Trp/Phe

\section{Figure 1}

Properties of the PA2794 locus. (A) Sequence predictions for PA2794. Shown are the results of structural prediction and sequence similarity searching techniques. The gene PA2794 is depicted 3 times, and shaded regions demonstrate distinct functional predictions. Regions are indicated using amino acid positions. Predicted transmembrane (TM) regions are shown first. These predictions were made using the programs TMPred (http://www.ch.embnet.org/software/TMPRED_ form.html) and TMAP (http://bioweb.pasteur.fr/seqanal/interfaces/ tmap.html). The second gene depiction shows the region most similar to other sialidase genes. Lighter gray shading indicates the region predicted by BLAST analysis. For an inclusive definition of the boundaries of this region, we used the most extreme amino- and carboxyterminal positions of BLAST alignments for the first 50 hits that were also labeled as sialidase or neuraminidase genes. The darker shading corresponds to the sialidase region predicted by the SMART program (http://smart.embl-heidelberg.de/). The third gene depiction shows the region similar to autotransporter genes. This region was determined by the BLAST alignment with a putative autotransporter (Magn03008734, GenBank accession no. ZP_00054112) from the Magnetospirillum magnetotacticum genome. This region had an $\mathrm{E}$ value score of $6 \times 10^{-4}$ in our BLAST search. (B) Predicted PAO1 neuraminidase amino acid sequence. ASP boxes are shaded gray.

\section{Results}

Construction of a nanA null mutant. Sequence predictions for the PA2794 locus were analyzed using ORFcurator (16), which identified both the sialidase region and a domain expected to have autotransporter function (Figure 1A) (17) consistent with previous reports (18). The predicted sequence included the ASP boxes expected to interact with sialic acid (Figure 1B) (19). An in-frame nonpolar deletion allele of the predicted neuraminidase open reading frame (PA2794, nanA) was constructed and used to replace the wild-type gene in PAO1 (Figure 2A). Loss of neuraminidase activity was documented by an assay monitoring the ability of culture supernatants to expose asialoGM1 from human airway epithelial cells (Figure 2B) (6). The deletion was shown not to impose any metabolic consequences on the fitness of the mutant, as growth curves of the wild-type, mutant, and complemented strains were comparable (Figure 2C).

Virulence properties of $\Delta 2794$ neuraminidase mutant differ depending upon the route of inoculation. To evaluate the role of the neuraminidase in respiratory tract infection, $2 \times 10^{8} \mathrm{CFU}$ inocula of the wild-type PAO1, $\Delta 2794$, and $\Delta 2794+$ nanA strains were used to

infect 7 - to 10 -day-old $\mathrm{BALB} / \mathrm{c}$ mice by the i.n. route, and morbidity and mortality were assessed 18 hours after infection (Figure 3 , A-D) (20). In contrast to PAO1, the $\Delta 2794$ mutant was readily cleared from the respiratory tract: $33 \%$ of the mice infected with $\Delta 2794$ developed pneumonia versus $78 \%$ of the mice infected with PAO1 $(P<0.05)$. Significantly fewer mice infected with the $\Delta 2794$ mutant became bacteremic: $10 \%$ versus $44 \%(P<0.05)$. Mortality rates were not significantly different (Figure 3A). Quantification of lung mRNA indicated less chemokine KC expression, which correlated with fewer polymorphonuclear leukocytes (PMNs) recruited to the lungs, in mice inoculated with $\Delta 2794$ compared with PAO1 $(P<0.05$; Figure 3, B and C). Histopathology similarly demonstrated substantially decreased inflammation in $\Delta 2794$ infected lungs (Figure 3D).

We also compared the virulence of wild-type and $\Delta 2794$ strains when introduced into mice by the i.p. route. This route of administration could potentially uncover differences in the immunogenicity of the mutant due to changes in glycosylation that might affect LPS or other surface structures. However, there were no differences in virulence when equal inocula of the wild-type and mutant strains were injected by the i.p. route (Figure 3E).

Immunostimulatory properties of the PA2794 mutant. To account for the attenuated phenotype of the $\Delta 2794$ mutant when introduced into the airways, we characterized its ability to attach to and stimulate chemokine and cytokine expression in both airway epithelial cells and macrophages (Figures 4 and 5). Although we had documented that PAO1 culture supernatant exposed more asialoGM1 on the surface of human airway cells than did $\Delta 2794$ (Figure 2B), there were no significant differences in either bacterial adherence or the induction of IL- 8 expression by the wildtype and $\Delta 2794$ mutant strains (Figure $4, \mathrm{~A}$ and B), in contrast to published reports that used concentrated purified enzyme (6). As bacterial modification of surface glycoconjugates could affect interactions with phagocytic cells, we compared the uptake and killing by RAW cells. Both wild-type and mutant bacteria were efficiently phagocytosed by RAW cells and both induced equivalent amounts of TNF- $\alpha$ production (Figure 5, A and B). In addition, we compared outer membrane proteins (OMPs) and secreted exoproducts from the wild-type and mutant strains and detected no differences (data not shown).

Effects of the PA2794 neuraminidase locus on LPS. Having found no biologically important alteration in eukaryotic surface glycosylation that could be attributed to effects of the neuraminidase, we next examined possible effects on surface structures including LPS. An extensive biochemical analysis of $P$. aeruginosa LPS had previously failed to identify sialic acid (15), in contrast to other neuraminidase-producing organisms such as Hemophilus (21). Although the enzyme could target a different amino sugar involved in LPS biosynthesis, a GC-mass spectroscopic analysis kindly performed by R. Ernst (University of Washington, Seattle, Washington, USA) did not reveal any differences in LPS lipid A structures among the wild-type, mutant, or complemented strains (data not shown) (22). As LPS structure is responsible for the resistance of $P$. aeruginosa to the lytic affects of normal human complement, we compared PAO1 and $\Delta 2794$ sensitivity to $10 \%$ human serum and also found no differences (data not shown). These negative results, along with the observation that the wildtype and mutant strains were equally virulent when injected i.p., indicate that the $\Delta 2794$ mutation does not have a major effect on LPS or its immunogenicity. 

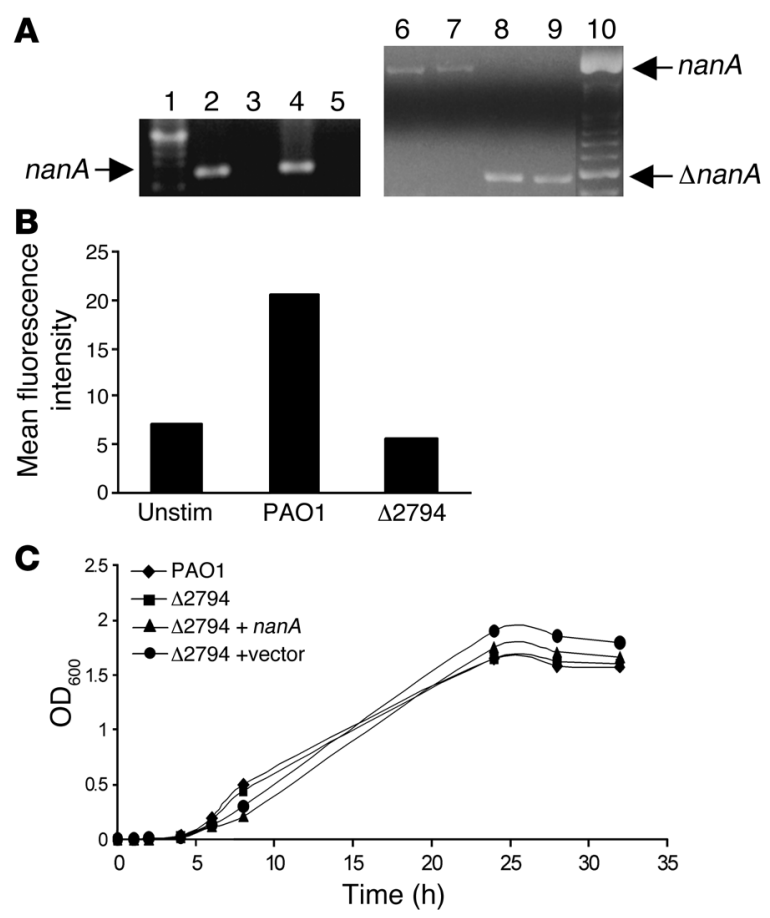

The PA2794 locus affects biofilm formation. Since the $\Delta 2794$ mutant appeared to be deficient solely in its ability to initiate infection by the mucosal route, we postulated that the neuraminidase might target other bacterial exopolysaccharides, such as those involved in biofilm formation. Bacteria-bacteria interactions were examined using crystal violet staining to quantify biofilm production in 96-well plates (Figure 6A) (23). The wild-type strain PAO1 containing an empty vector was used as a control, and all strains were grown under identical conditions in gentamicin selection. The $\Delta 2794$ mutant with the empty vector produced significantly less biofilm than PAO1 also containing the control vector $(P<0.001)$. Biofilm production by the $\Delta 2794$ mutant expressing the cloned nanA locus was significantly greater than that of the parental strain $(P<0.0001)$, consistent with the constitutive expression of the cloned gene. Similarly, overexpression of nanA by expressing the cloned gene in PAO1 also resulted in significantly greater biofilm production $(P<0.0001)$. In addition, we tested an independently derived mutation in strain PAK and a $\Delta 2794$ PAK mutant. Although PAK did not produce significant amounts of biofilm under control conditions, and deletion of the 2794 locus had no apparent effect, overexpression of the cloned 2794 locus in $\Delta 2794$ PAK resulted in a significant increase in the production of biofilm compared with the PAK parental strain $(P<0.0001)$. Biofilm assays were then performed in a flow cell (Figure 6C) (24) and on a rotating disc reactor (Figure 6D) (25) in order to formally evaluate the ability of the bacteria to form structured communities. In each assay system, the biofilm produced by $\Delta 2794$ exhibited dramatically changed architecture, and complementation of the phenotype by the cloned gene was observed. To establish that the mutant $\Delta 2794$ exhibits similarly impaired biofilm formation on epithelial cells, we incubated GFP-expressing bacteria with monolayers of airway epithelial cells and observed by fluorescence imaging that the $\Delta 2794$ organisms did not cluster or autoagglutinate, in contrast to PAO1 (Figure 6E).

\section{Figure 2}

Characterization of the $\triangle 2794$ mutant. (A) Colony PCR for expression of nanA. Lanes $2-5$ show PCR using primers from within the nanA gene; lanes 6-9 show PCR using primers flanking nanA. Lane 2, PAO1; lane 3, $\Delta 2794$; lane 4, $\Delta 2794$ + nanA; lane 5, $\Delta 2794$ with vector alone; lane 6, PAO1; lane 7, PAK; lane 8, $\Delta 2794$; lane 9, $\triangle 2794$ PAK. Lanes 1 and 10 show molecular weight markers. (B) Amount of superficial asialoGM1 on $16 \mathrm{HBE}$ cells following exposure to bacterial supernatant from the indicated strains was quantified by flow cytometry. A representative experiment is shown. Unstim, unstimulated (i.e., treated with media alone). (C) Growth of strains in M9 media as determined by $\mathrm{OD}_{600}$. A representative experiment is shown.

Neuraminidase inbibitors block biofilm production. Drugs that target the neuraminidase produced by influenza viruses are an important component of antiviral chemotherapy, used for both prophylaxis and treatment. As bacterial and viral neuraminidases can share common ASP boxes that interact with sialic acid (19), we postulated that the neuraminidase inhibitors designed to block the influenza enzyme (26) might have sufficient avidity for the active site of the bacterial neuraminidase to inhibit biofilm formation. We tested the effects of the influenza virus neuraminidase inhibitors oseltamivir (27) and peramivir (28) on PAO1 biofilm formation using the crystal violet assay (Figure 7, A and B). A dose-dependent effect was observed with each of the 2 drugs, suggesting that $P$. aeruginosa biofilm formation may be a target to prevent infections in patients at risk by using neuraminidase inhibitors.

To document that these viral neuraminidase inhibitors are specifically interacting with the bacterial neuraminidase, we also tested their effect in blocking neuraminidase activity using the fluorescent substrate 2 '-(4-methylumbelliferyl)- $\alpha$-D- $N$-acetylneuraminic acid (Figure $7 \mathrm{C}$ ). The $V$. cholerae enzyme as well as the $P$. aeruginosa $\mathrm{PAO} 1$ enzyme were inhibited by peramivir, suggesting that the active sites of both types of neuraminidases have some affinity for the available viral neuraminidase inhibitors.

\section{Discussion}

Bacterial neuraminidases have long been implicated in the pathogenesis of mucosal infection. Their prevalence and conservation among a very broad range of important human pathogens such as $S$. pneumoniae and $V$. cholerae as well as commensal flora, Mycoplasma (29), and even the gut flora of fish (30) implies a critical role in microbial ecology, which may vary according to bacterial species and sites of infection (1). The contribution of neuraminidase expression to the pathogenesis of respiratory infection is complex. The expected analyses of isogenic neuraminidase mutants has been accomplished in few bacterial species, due to gene redundancies $(31,32)$, involvement in metabolic pathways, and perhaps technical issues, as the construction of mutants has been unexpectedly difficult.

Initial studies of the $P$. aeruginosa neuraminidase performed with purified enzyme and in vitro analyses were entirely consistent with a role for the enzyme in modifying airway epithelial cell surfaces to facilitate bacterial attachment (6). Moreover, as CF airways were more readily modified than were normal airway cells (11), the Pseudomonas enzyme seemed likely to be important in that disease. However, in the studies presented herein, performed under more physiological conditions in vivo using isogenic mutants, we now find an entirely different function for the $P$. aeruginosa neuraminidase. The neuraminidase appears to 

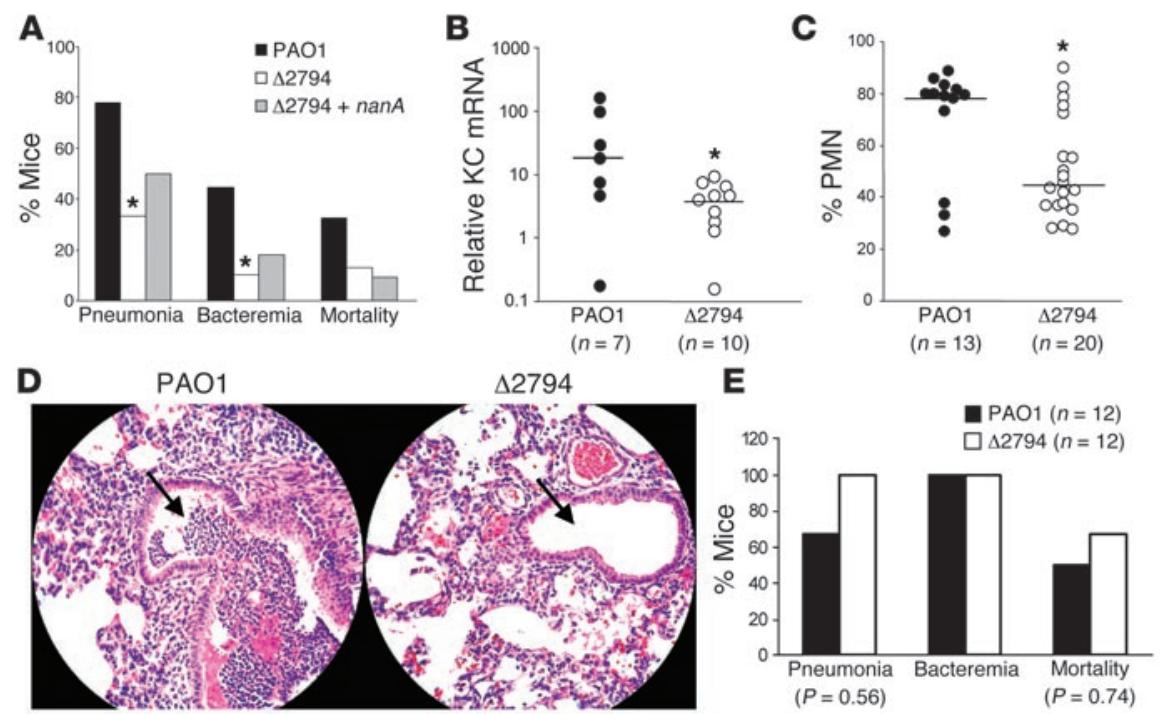

Figure 3

Comparison of PAO1 and $\Delta 2794$ virulence. (A-D) Mouse model of pneumonia. (A) The percentage of the total number of mice inoculated i.n. with each strain that developed pneumonia or bacteremia or died is indicated. (B) KC mRNA expression was determined by real-time PCR and standardized to actin. Individual mouse values are shown; horizontal lines indicate the median value of each group. (C) Lung cell suspensions from mice inoculated with $P$. aeruginosa PAO1 or $\triangle 2794$ were stained for CD45 and Ly6G (i.e., PMNs). Leukocytes were gated based on CD45 expression, and the percentage of Ly6G-positive cells was determined. Individual mouse values are shown; horizontal lines indicate the median value of each group. ${ }^{\star} P<0.05$, Fisher's exact test $(\mathbf{A})$; nonparametric Mann-Whitney test (B and C). (D) H\&Estained sections of murine lungs 16 hours following inoculation with $P$. aeruginosa PAO1 or $\Delta 2794$. Arrows demonstrate that airways are full of PMNs in PAO1-infected lung but clear in $\triangle 2794$-infected lung. (E) Comparison of PAO1 and $\triangle 2794$ virulence when introduced i.p. BALB/c mice (7-10 days old) were inoculated i.p. with PAO1 or $\Delta 2794$ ( $n=12$ per group). Mice were euthanized 16 hours later, and lungs and spleens were harvested for plating of single-cell suspensions. The percentage of mice inoculated with each strain that developed pneumonia or bacteremia or died is indicated. Pneumonia was defined as the recovery of more than 1,000 CFU per lung; bacteremia was defined as the presence of bacteria in the spleen.

be important for biofilm production, the cell-cell interactions which are critical even in the initial colonization process. Exactly how the PA2794 neuraminidase is involved in biofilm synthesis is unclear. Although the components of the PAO1 biofilm have not been fully defined, those of another $P$. aeruginosa, PA14, do not include sialic acid (33), indicating that the neuraminidase activity may be directed against a different sugar linkage on the bacterial surface. Pseudaminic acid - or a structure containing pseudaminic acid, a 9-carbon acidic sugar with structural similarity to the neuraminic acids - is a potential substrate and modifies several surface structures including LPS (34), pili (35), and flagella (36) in $P$. aeruginosa. Recent studies indicate that there are significant homologies among the genes involved in sialic acid O-acetylation in many bacterial species including $P$. aeruginosa strain 012 , which produces pseudaminic acid but not sialic acid (37). Pseudaminic acid is involved in the glycosylation of Campylobacter pylori flagella (38), and mutants that lack pseudaminic acid fail to autoagglutinate (as we found in the $\Delta 2794$ mutant) and are attenuated in a ferret model of diarrheal disease. Mutations in the biosynthetic pathway involved in the addition of pseudaminic acid to flagella appear to contribute to this phenotype. Just as autolysins are necessary for cell wall biosynthesis, enzymes capable of cleaving carbohydrate linkages are necessary for the growth and modificalogically significant consequence of the loss of neuraminidase appears to be limited to its defect in cell-cell aggregation.

The importance of biofilm production in the pathogenesis of respiratory tract infection caused by other common respiratory pathogens such as $H$. influenzae $(21,44)$ and $S$. pneumoniae has been well described (45). Less clear is whether these biofilms require the participation of neuraminidases for biosynthesis or modification. H. influenzae are known to produce sialylated biofilms that are important in the pathogenesis of mucosal infections, such as otitis media (46). While neuraminidase production by Hemophilus species has been reported (47), its specific involvement in colonization or pathogenesis has not yet been established. Several functions have been ascribed to the pneumococcal neuraminidase(s), which can desialylate host proteins (5) as well as surface components from adjacent flora (48) but does not appear to be critical for invasive infection (49), as was the case for the Pseudomonas enzyme. The surface location of the pneumococcal enzyme is consistent with a role in biofilm biology (4) but more definitive, functional experiments are necessary.

The mucosal surface of the gut is also colonized by organisms in biofilms (50), and neuraminidase expression has been well characterized in gastrointestinal pathogens (50) including Salmonellae (51), Vibrios (52), and Bacteroides (8). Neuraminidases 

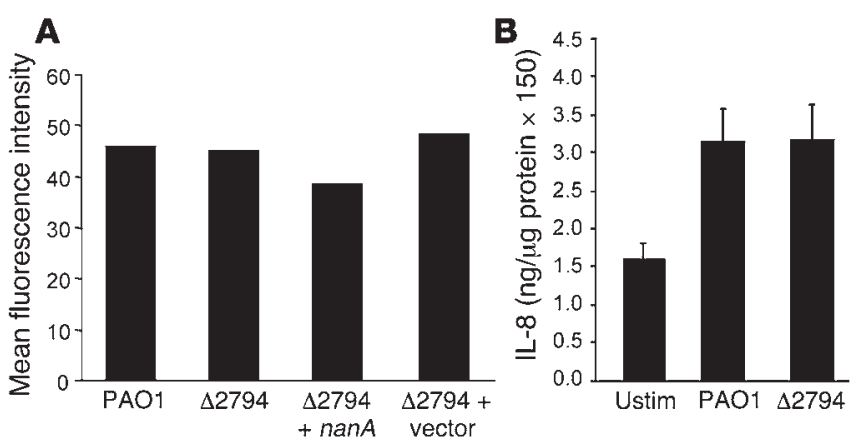

Figure 4

Interactions of PAO1 and $\Delta 2794$ with human airway epithelial cells. (A) Bacterial adherence following a 1-hour exposure to $16 \mathrm{HBE}$ airway epithelial cells was determined by flow cytometry. Cells were stained with polyclonal anti-OMP followed by Alexa Fluor 488-conjugated anti-rabbit IgG. The number of bacteria bound to the surface was quantitated as mean fluorescence intensity. (B) Induction of IL-8 production by confluent monolayers of $1 \mathrm{HAE}$ airway epithelial cells following exposure to bacteria - compared with induction by bacterial growth media alone as a control - was quantified by ELISA as previously described (57).

contribute to bacterial metabolism in some of these organisms, and bacterial surface sialylation may provide an immune masking function as well (3). However, even though neuraminidase expression by $V$. cholerae has been characterized for 40 years (52), its specific contribution to the pathogenesis of cholera remains obscure (53). Although cholera toxin targets sialic acid, it has never been clearly established how the neuraminidase is linked to the toxin (53) or if the enzyme is also involved in cell-cell interactions and the colonization process. There may be technical issues that have limited the analysis of cloned genes that contribute to biofilm formation, as it has only recently been recognized that the TEM $\beta$-lactamase often used for selection interferes with biofilm formation (54).

There has been great interest in identifying bacterial gene products that are essential for pathogenesis, as these could be targeted in the patient populations known to be at high risk for infection. This is an especially appealing strategy to prevent $P$. aeruginosa infection in CF and intensive care patients. Viral neuraminidase inhibitors have been very useful in the prevention and treatment of influenza, targeting similar high-risk patient populations. The PA2794 neuraminidase shares many conserved elements and folds in the manner predicted for other microbial neuraminidases (19, 55). A preliminary analysis of PA2794 crystal structure indicates that the enzyme shares the same sialic acid binding fold as does the influenza enzyme, but otherwise has little homology (L. Tong

\section{Figure 5}

Interactions of PAO1 and $\Delta 2794$ with phagocytic cells. (A) Binding and phagocytosis of PAO1 and $\triangle 2794$ by RAW cells was determined by flow cytometry. Adherent organisms were quantified by staining with anti-OMP, and adherent plus internalized bacteria were quantified in RAW cells that were permeabilized prior to staining to determine total RAW cell-associated bacteria. (B) TNF- $\alpha$ production by RAW cells treated with media alone, bacterial culture supernatant, or LPS harvested from cultures of PAO1 and $\Delta 2794$ was quantified by ELISA. Purified LPS (Sigma-Aldrich) was used as a positive control. and Y.-S. Hsiao, unpublished observations). The activity of the viral neuraminidase inhibitors in blocking $P$. aeruginosa biofilm production in vitro suggests that bacterial neuraminidases may also be a useful target to prevent infection in patients at high risk. These or similar compounds optimized for activity against bacterial enzymes could be especially useful in preventing $P$. aeruginosa colonization in CF patients. As neuraminidase expression is so highly conserved in mucosal pathogens, it should be possible to determine whether this approach blocks bacterial biofilm production and infection by other common pathogens.

\section{Methods}

Construction of a $P$. aeruginosa PAO1 neuraminidase null mutant. A nanA null mutant $(\Delta 2794)$ was constructed by allelic replacement. An in-frame nonpolar deletion allele was constructed by removing the nan $A$ coding sequence corresponding to amino acids 5-435 of the predicted 438-residue polypeptide and used to replace the full-length gene (1,317 base pairs) by the method previously described (56). Primers were designed using the published DNA sequence for the neuraminidase gene (designated PA2794) from $P$. aeruginosa strain PAO1 (GenBank accession no. AF236853). A nanA complementation plasmid was constructed by cloning a PCR product corresponding to the full-length neuraminidase open reading frame into plasmid $\mathrm{PMMBGW}$ with either a gentamicin or a penicillin resistance marker (56). The complementation clone or an empty vector control was introduced into the $\Delta 2794$ mutant by conjugation and selection on gentamicin $(40 \mu \mathrm{g} / \mathrm{ml})$ or piperacillin $(100 \mu \mathrm{g} / \mathrm{ml})$. The same procedure was carried out to generate a $\Delta 2794$ mutation in the P. aeruginosa strain PAK. The following primers were used for genotyping: internal to PA2794, 5'CGCACTATACACAGGAACACG-3' and $5^{\prime}$-GCCTAGCGGAAGGATCGTCGC-3'; external to PA2794, 5'-GATTATAAGTCTGCCGTCGG-3' and 5'-CTCGGGAAACGTGCACATCC-3'.

Bacterial strains and culture conditions. The standard laboratory strain of P. aeruginosa PAO1 was used as a prototype, grown in Luria broth (LB) or M9 media with Mg-glu as indicated. For complementation studies, the PA2794 locus was overexpressed in E. coli using pMMB67EH.gm and pMMB67EH.amp. Growth curves were obtained by growing bacteria in M9 media - with $40 \mu \mathrm{g} / \mathrm{ml}$ gentamicin or $100 \mu \mathrm{g} / \mathrm{ml}$ of piperacillin selection for strains containing plasmid - overnight to stationary phase, then
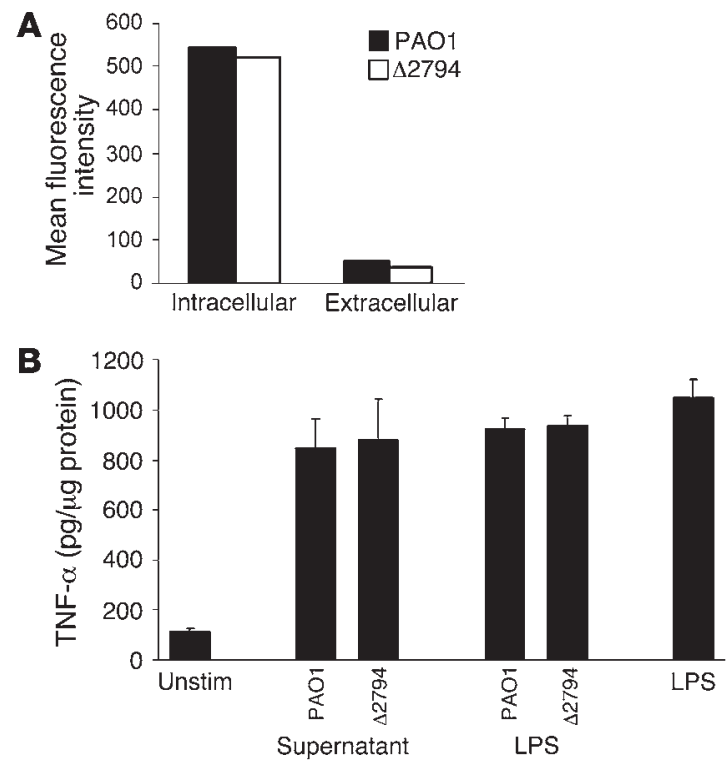
A

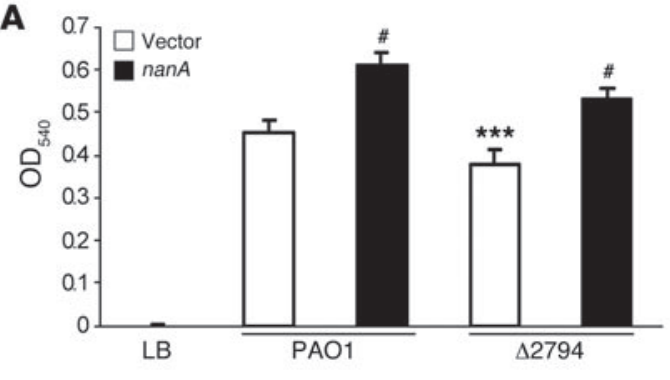

C
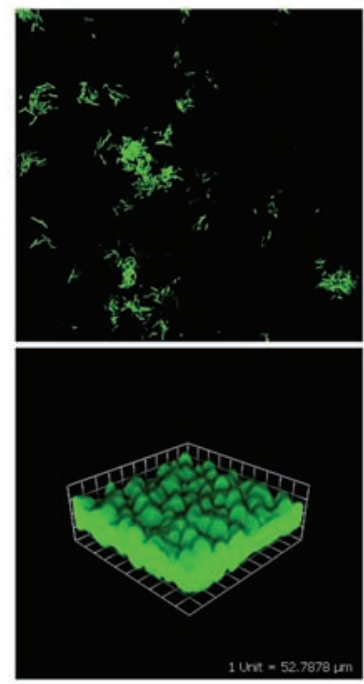

D

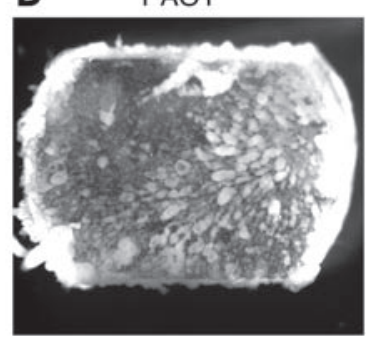

$\triangle 2794$
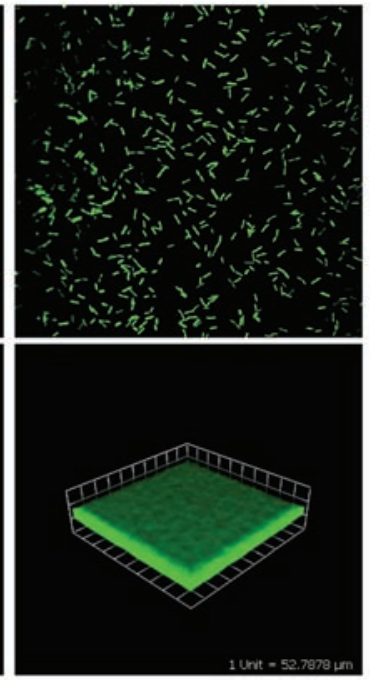

$\Delta 2794$

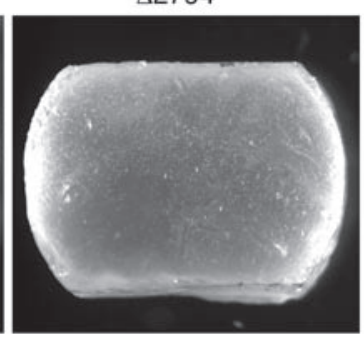

B

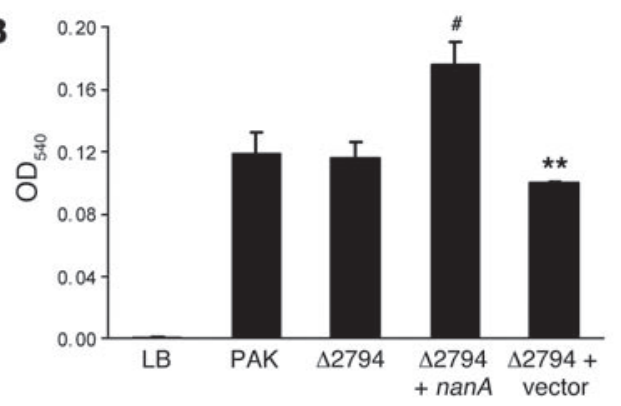

$\Delta 2794+\operatorname{nan} A$

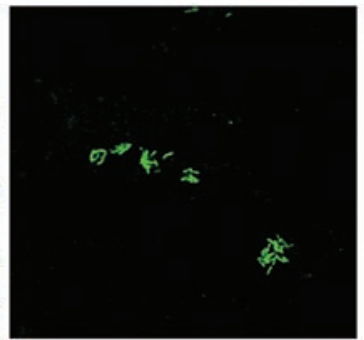

$\Delta 2794+$ vector
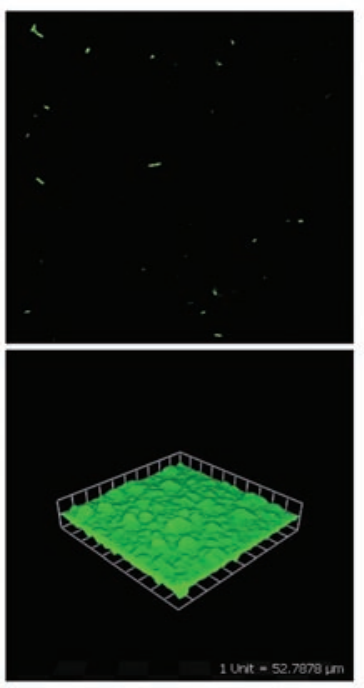

$\Delta 2794$

E

$\mathrm{PAO} 1$
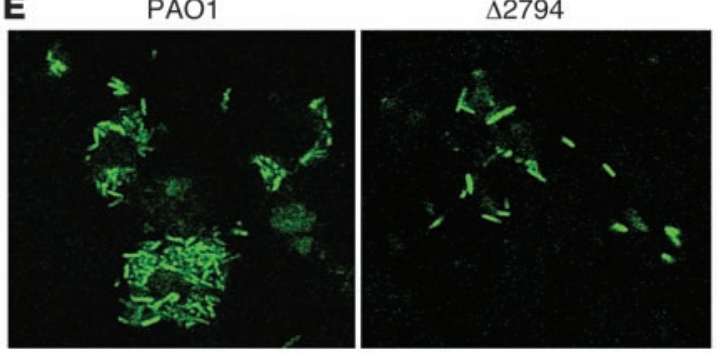

\section{Figure 6}

Comparison of biofilm production by PAO1 and $\Delta 2794$. (A and B) Biofilm production by bacterial strains detected by crystal violet staining. (A) PAO1 and $\Delta 2794$ each with either empty control vector (white bars) or cloned nanA (black bars) and (B) PAK, $\triangle 2794$ PAK, $\triangle 2794$ PAK + neuraminidase locus $(\Delta 2794+$ nanA $)$, and $\Delta 2794$ PAK plus empty control vector are shown. ${ }^{\star \star} P<0.01,{ }^{\star \star *} P<0.001$, ${ }^{*} P<0.0001$ versus PAO1 + vector (A) or PAK (B). (C) Fluorescence microscope images (top row) and profiles (bottom row) of biofilm production in a flow cell. Compared with PAO1, $\Delta 2794$ formed less differentiated and flattened layers. The $\Delta 2794+$ nanA complemented mutant showed restored, differentiated clumps of cells, while the $\Delta 2794$ mutant plus control vector did not. (D) Light microscope images of biofilms produced under more dynamic conditions using a rotating disc reactor that uses removable discs attached to a rotor in a chemostat. The disc rotation produces high-shear forces. High-shear conditions can accentuate deficiencies in attachment and matrix integrity. White, fluffy material is biofilm. The lack of biofilm formation by the mutant could be caused by defective matrix synthesis. (E) Bacteria expressing GFP $\left(10^{6}\right)$ were incubated with confluent monolayers of $16 \mathrm{HBE}$ airway epithelial cells for 5 hours and visualized by confocal microscopy.

diluting $1: 1,000$ in fresh media and incubating at $37^{\circ} \mathrm{C}$ with shaking and $\mathrm{OD}_{600}$ readings taken over time.

Epithelial cell culture. Originally obtained from D. Gruenert (California Pacific Medical Center Research Institute, San Francisco, California, USA), $1 \mathrm{HAE}$ and $16 \mathrm{HBE}$ cells were grown in minimum essential medium with Earle's salts supplemented with $10 \%$ fetal calf serum (Molecular Probes) as previously described (57). RAW cells were grown in RPMI medium 1640 with $10 \%$ fetal calf serum.
Biofilm assays. An overnight culture of bacteria grown in LB with shaking was diluted $1: 100$, and $100-\mu \mathrm{l}$ aliquots added to 96 -well microtiter plates were incubated for $24-48$ hours at $37^{\circ} \mathrm{C}(58)$. Crystal violet $(0.1 \%)$ was added to each well for 15 minutes, rinsed 3 times with water, and then released with the addition of $200 \mu \mathrm{l}$ of $95 \%$ ethanol. Absorbance was determined at $540 \mathrm{~nm}$. For experiments with inhibitors, the following modifications in the assay were used: for oseltamivir, an overnight culture of bacteria was diluted 1:100 in different doses of inhibitor in LB, and $100 \mu \mathrm{l}$ 

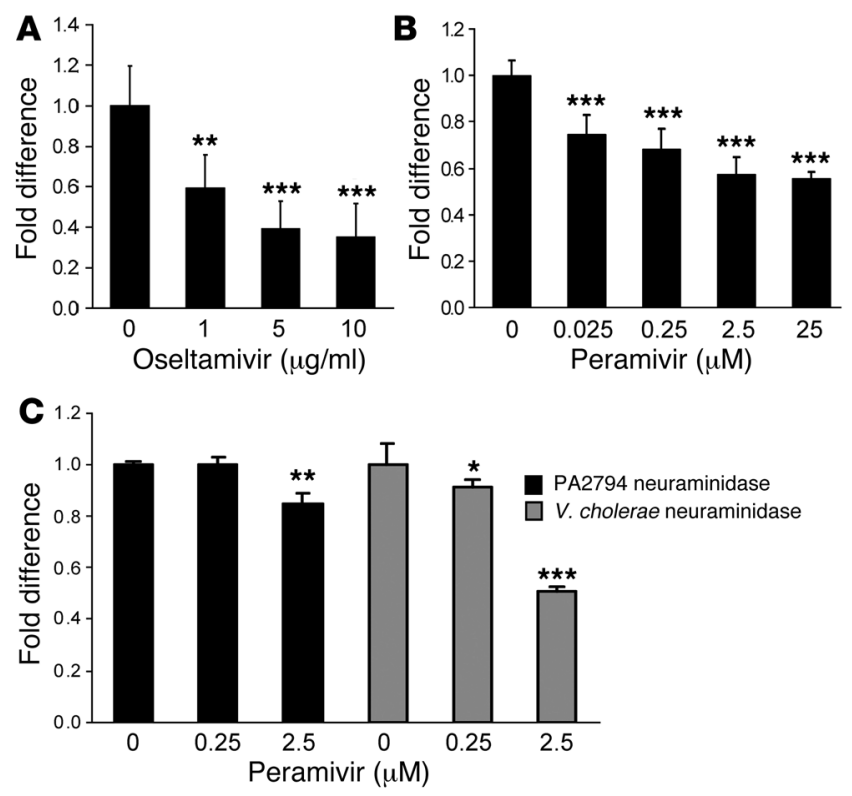

aliquots were plated and assayed as described above; for peramivir, an overnight culture of bacteria was diluted 1:100 in different doses of inhibitor in $\mathrm{LB}$, incubated at room temperature for 48 hours, and then diluted 1:2 in $\mathrm{LB}$, and $100 \mu \mathrm{l}$ aliquots were plated and assayed as described above. Each sample was tested in sextuplicate, the assay was repeated on 3 separate occasions, and representative data are shown. For the complementation studies, all of the PAO1 strains were transformed with either the control vector or the vector expressing the 2794 locus. Flow cell experiments and confocal microscopy were performed as previously described (24). For visualization by confocal microscopy, pMRP9-1 (which expresses GFP) was transformed into appropriate strains (59). The rotating disk reactor (25) was used for generating biofilms for microscopy and quantitative counts. Tryptic soy broth medium (1:100 strength) was used for these experiments.

Neuraminidase assays. The PAO1 enzyme $(10 \mu \mathrm{g} / \mathrm{ml})$ was overexpressed and purified from E. coli using the pET28a vector (Novagen, EMD Biosciences), and a control V. cholera neuraminidase $(0.1 \mathrm{U} / \mathrm{ml}$; Calbiochem, EMD Biosciences) was incubated with the fluorescent substrate 2 '-(4-methylumbelliferyl)- $\alpha$-D- $N$-acetylneuraminic acid (Sigma-Aldrich) $(25 \mu \mathrm{M}$ in $0.9 \% \mathrm{NaCl})$ with or without peramivir $(0.25$ and $2.5 \mu \mathrm{M})$ for 24-48 hours, and fluorescence was read at excitation $360 \mathrm{~nm}$ and emission $465 \mathrm{~nm}$. Each data point was performed in sextuplicate. A representative experiment is shown.

IL-8 assays. Confluent monolayers of 1 HAEo cells, weaned from serum overnight, were washed and stimulated with bacteria $\left(1 \times 10^{8} \mathrm{CFU} / \mathrm{ml}\right)$ for 30 minutes. Fresh media plus gentamicin $(100 \mu \mathrm{g} / \mathrm{ml})$ was added and then removed for chemokine analysis after 3 hours. ELISA for IL-8 (R\&D Systems) was performed as previously described (56). Each data point was performed in quintuplicate and standardized by protein. Each experiment was performed at least 3 times, and a representative study is shown.

Quantification of epithelial sialylation by flow cytometry. 16HBE cells were grown in 24-well plates to confluence and exposed to bacterial supernatant concentrated 30 -fold for 3-5 hours followed by 3 PBS washes. Cells were stained with rabbit polyclonal Anti asialo GM1 antibody (Wako) followed by Alexa Fluor 488 donkey anti-rabbit IgG (Molecular Probes). Cells detached from the plastic using $0.02 \%$ EGTA in HBSS were then fixed with $1 \%$ paraformaldehyde and analyzed on a FACSCalibur using CellQuest software (version $3.3 ; \mathrm{BD}$ ).

\section{Figure 7}

Dose-dependent inhibition of biofilm production in response to viral neuraminidase inhibitors. PAO1 was grown in the presence of indicated concentrations of (A) oseltamivir and (B) peramivir before plating for assay, and biofilm production was detected by crystal violet staining method. Fold difference compared with untreated is plotted. A representative experiment is shown. (C) Inhibition of bacterial neuraminidases by peramivir. Purified PAO1 neuraminidase and $V$. cholerae neuraminidase were incubated with and without peramivir, and neuraminidase activity was measured using the fluorescent substrate 2 '-(4-methylumbelliferyl)- $\alpha-D-N$-acetylneuraminic acid. Fold difference compared with untreated is plotted. A representative experiment is shown. ${ }^{*} P<0.05,{ }^{* \star} P<0.01,{ }^{* \star} P<0.001$ versus untreated.
RAW cell binding and phagocytosis as determined by flow cytometry. RAW cells, a murine monocyte-macrophage cell line, were grown in 10-cm dishes and exposed to $1 \times 10^{8}$ bacteria for 30 minutes at $37^{\circ} \mathrm{C}$. After 4 washes with PBS, $2 \mathrm{ml}$ HBSS plus $0.02 \%$ EGTA was added, and cells were harvested. Cells were counted in a hemacytometer, and $1 \times 10^{6}$ cells were aliquoted per microfuge tube. PBS $(1 \mathrm{ml})$ was added to each tube, and cells were pelleted at $400 \mathrm{~g}$ for 5 minutes. For extracellular binding determination, cells were incubated in 5\% normal serum in PBS. For determination of total external and internalized bacteria, cells were incubated with Perm/Wash buffer (BD Biosciences - Pharmingen). In both cases, cells were then stained with rabbit anti-OMP antibody followed by Alexa Fluor 488 donkey anti-rabbit secondary and fixed with $1 \%$ paraformaldehyde and analyzed on a FACSCalibur using CellQuest software (version 3.3; BD).

Mouse models of infection. Mouse protocol number AAAA1718 was approved by the Institutional Animal Care and Use Committee at Columbia University. Seven day old BALB/c mice were inoculated i.n. with $2 \times 10^{8}$ CFU of PAO1 or $\triangle 2794$ in $10 \mu$ of PBS or i.p. with $5 \times 10^{5} \mathrm{CFU}$ of PAO1 or $\triangle 2794$ and euthanized 16 hours later with pentobarbital. Pneumonia was defined as the recovery of more than 1,000 CFU per lung, and bacteremia was defined as the recovery of bacteria from the spleen. The inflammatory response in vivo was assayed by flow cytometry as previously described (60). Single-cell suspensions of the lung were screened for the percentage of PMNs in the total leukocyte population by double staining with PE-labeled anti-CD45 and FITC-labeled anti-Ly6G antibodies (BD Biosciences - Pharmingen). Irrelevant, isotype-matched antibodies were used as a control. Cells were gated on the basis of their forward and side scatter profiles and analyzed for the expression of both CD45 and Ly6G.

Immunohistochemistry. Paraffin lung sections from mice infected with PAO1 and $\triangle 2794$ were stained with $\mathrm{H} \& \mathrm{E}$.

Real-time PCR. Lungs from PAO1- and $\Delta 2794$-inoculated mice were obtained 16-18 hours after inoculation and stored in RNAlater (QIAGEN). RNA was isolated using the QIAGEN RNeasy Mini Kit. cDNA was made from $1 \mu \mathrm{g}$ of RNA using the iScript cDNA Synthesis Kit (Bio-Rad). For quantitative real-time PCR, amplification was performed in a LightCycler using the DNA Master SYBR Green I kit (Roche Diagnostics). Primers used for KC amplification were 5'-CCGCGCCTATCGCCAATGAGCTGCGC-3' and 5'-CTTGGGGACACCTTTTAGCATCTTTTGG-3', and 35 cycles were 
run with denaturation at $95^{\circ} \mathrm{C}$ for 8 seconds, amplification at $56^{\circ} \mathrm{C}$ for 10 seconds, and extension at $72^{\circ} \mathrm{C}$ for 12 seconds. Actin was amplified on each individual sample and used as control for standardization. Primers used for actin amplification were 5'-GTGGGGCGCCCCAGGCACCA-3' and $5^{\prime}$-CGGTTGGCCTTGGGGTTCAGGGGGG-3', and 35 cycles were run with denaturation at $95^{\circ} \mathrm{C}$ for 8 seconds, amplification at $63^{\circ} \mathrm{C}$ for 10 seconds, and extension at $72^{\circ} \mathrm{C}$ for 12 seconds.

Adherence assay. 16HBE airway epithelial cells were stimulated with bacteria for 1 hour. After washing with PBS to remove unbound organisms, cells were stained with polyclonal anti-OMP followed by Alexa Fluor 488-conjugated anti-rabbit IgG (Molecular Probes). Fixed cells were analyzed by flow cytometry to quantitate the number of bacteria bound to the surface.

Statistics. For biofilm, neuraminidase, and IL-8 assays, means and standard deviations were calculated, and statistical significance was determined using 2-tailed unpaired Student's $t$ test (biofilm assays) and 1-way analysis of variance with Bonferroni's post test (neuraminidase and IL-8 assays).
Tests of statistical significance were performed with GraphPad InStat (version 3.0; GraphPad Software).

\section{Acknowledgments}

This work was funded by NIH grant R01 DK39693. M.I. Gomez was supported by a Cystic Fibrosis Foundation postdoctoral fellowship. We thank George Drusano and James MeSharry for providing peramivir and Stephen Lory for his help in constructing the mutants.

Received for publication January 13, 2006, and accepted in revised form May 23, 2006.

Address correspondence to: Alice Prince, Columbia University, 650 West 168th Street, BB 4-416, New York, New York 10032, USA. Phone: (212) 305-4193; Fax: (212) 342-5728; E-mail: asp7@columbia.edu.
1. Vimr, E.R., Kalivoda, K.A., Deszo, E.L., and Steenbergen, S.M. 2004. Diversity of microbial sialic acid metabolism. Microbiol. Mol. Biol. Rev. 68:132-153.

2. Colman, P.M. 1994. Influenza virus neuraminidase: structure, antibodies, and inhibitors. Protein Sci. 3:1687-1696.

3. Vimr, E., and Lichtensteiger, C. 2002. To sialylate, or not to sialylate: that is the question. Trends Microbiol. 10:254-257.

4. Camara, M., Boulnois, G.J., Andrew, P.W., and Mitchell, T.J. 1994. A neuraminidase from Streptococcus pneumoniae has the features of a surface protein. Infect. Immun. 62:3688-3695.

5. King, S.J., et al. 2004. Phase variable desialylation of host proteins that bind to Streptococcus pneumoniae in vivo and protect the airway. Mol. Microbiol. 54:159-171.

6. Cacalano, G., Kays, M., Saiman, L., and Prince, A. 1992. Production of the Pseudomonas aeruginosa neuraminidase is increased under hyperosmolar conditions and is regulated by genes involved in alginate expression. J. Clin. Invest. 89:1866-1874.

7. Krivan, H.C., Roberts, D.D., and Ginsburg, V. 1988. Many pulmonary pathogenic bacteria bind specifically to the carbohydrate sequence GalNAc $\beta 1-4 \mathrm{Gal}$ found in some glycolipids. Proc. Natl. Acad. Sci.U.S. A. 85:6157-6161.

8. Godoy, V.G., Dallas, M.M., Russo, T.A., and Malamy, M.H. 1993. A role for Bacteroides fragilis neuraminidase in bacterial growth in two model systems. Infect. Immun. 61:4415-4426.

9. Bouchet, V., et al. 2003. Host-derived sialic acid is incorporated into Haemophilus influenzae lipopolysaccharide and is a major virulence factor in experimental otitis media. Proc. Natl. Acad. Sci. U. S. A. 100:8898-8903.

10. Leprat, R., and Michel-Briand, Y. 1980. Extracellular neuraminidase production by a strain of Psendomonas aeruginosa isolated from cystic fibrosis. Ann. Microbiol. 131B:209-222.

11. Saiman, L., and Prince, A. 1993. Pseudomonas aeruginosa pili bind to asialoGM1 which is increased on the surface of cystic fibrosis epithelial cells. J. Clin. Invest. 92:1875-1880.

12. Baltimore, R.S., Christie, C.D., and Smith, G.J. 1989. Immunohistopathologic localization of Psendomonas aeruginosa in lungs from patients with cystic fibrosis. Implications for the pathogenesis of progressive lung deterioration. Am. Rev. Respir. Dis. 140:1650-1661.

13. Boucher, R.C. 2004. New concepts of the pathogenesis of cystic fibrosis lung disease. Eur. Respir. J. 23:146-158.

14. Lanotte, P., et al. 2004. Genetic features of Pseudomonas aeruginosa isolates from cystic fibrosis patients compared with those of isolates from other origins. J. Med. Microbiol. 53:73-81.

15. Knirel Yu, A., et al. 1988. The structure of O-specific polysaccharides and serological classification of Pseudomonas aeruginosa (a review). Acta Microbiol. Hung. 35:3-24.

16. Rosenfeld, J.A., Sarkar, I.N., Planet, P.J., Figurski, D.H., and DeSalle, R. 2004. ORFcurator: molecular curation of genes and gene clusters in prokaryotic organisms. Bioinformatics. 20:3462-3465.

17. Henderson, I.R., Navarro-Garcia, F., Desvaux, M. Fernandez, R.C., and Ala'Aldeen, D. 2004. Type V protein secretion pathway: the autotransporter story. Microbiol. Mol. Biol. Rev. 68:692-744.

18. Corfield, A.P., Veh, R.W., Wember, M., Michalski, J.C., and Schauer, R. 1981. The release of $\mathrm{N}$-acetyland $\mathrm{N}$-glycolloyl-neuraminic acid from soluble complex carbohydrates and erythrocytes by bacterial, viral and mammalian sialidases. Biochem. J. 197:293-299.

19. Roggentin, P., et al. 1989. Conserved sequences in bacterial and viral sialidases. Glycoconj. J. 6:349-353.

20. Tang, H.B., et al. 1996. Contribution of specific Psendomonas aeruginosa virulence factors to pathogenesis of pneumonia in a neonatal mouse model of infection. Infect. Immun. 64:37-43.

21. Swords, W.E., et al. 2004. Sialylation of lipooligosaccharides promotes biofilm formation by nontypeable Haemophilus influenzae. Infect. Immun. 72:106-113.

22. Ernst, R.K., et al. 1999. Specific lipopolysaccharide found in cystic fibrosis airway Pseudomonas aeruginosa. Science. 286:1561-1565.

23. O’Toole, G., Kaplan, H.B., and Kolter, R. 2000. Biofilm formation as microbial development. Annu. Rev. Microbiol. 54:49-79.

24. Boles, B.R., Thoendel, M., and Singh, P.K. 2004 Self-generated diversity produces "insurance effects" in biofilm communities. Proc. Natl. Acad. Sci.U. S. A. 101:16630-16635.

25. Singh, P.K., Parsek, M.R., Greenberg, E.P., and Welsh, M.J. 2002. A component of innate immunity prevents bacterial biofilm development. Nature. 417:552-555.

26. Hayden, F.G., et al. 1999. Use of the oral neuraminidase inhibitor oseltamivir in experimental human influenza: randomized controlled trials for prevention and treatment. JAMA. 282:1240-1246.

27. McKimm-Breschkin, J., et al. 2003. Neuraminidase sequence analysis and susceptibilities of influenza virus clinical isolates to zanamivir and oseltamivir. Antimicrob. Agents Chemother. 47:2264-2272.

28. Sidwell, R.W., and Smee, D.F. 2002. Peramivir (BCX1812, RWJ-270201): potential new therapy for influenza. Expert Opin. Investig. Drugs. 11:859-869.

29. Sethi, K.K., and Muller, H.E. 1972. Neuraminidase activity in Mycoplasma gallisepticum. Infect. Immun.
5:260-262.

30. Sugita, H., Shinagawa, Y., and Okano, R. 2000. Neuraminidase-producing ability of intestinal bacteria isolated from coastal fish. Lett. Appl. Microbiol. 31:10-13.

31. Berry, A.M., Lock, R.A., and Paton, J.C. 1996. Cloning and characterization of nanB, a second Streptococcus pneumoniae neuraminidase gene, and purification of the NanB enzyme from recombinant Escherichia coli. J. Bacteriol. 178:4854-4860.

32. Polissi, A., et al. 1998. Large-scale identification of virulence genes from Streptococcus pneumoniae. Infect. Immun. 66:5620-5629.

33. Wozniak, D.J., et al. 2003. Alginate is not a significant component of the extracellular polysaccharide matrix of PA14 and PAO1 Pseudomonas aeruginosa biofilms. Proc. Natl. Acad. Sci. U. S. A. 100:7907-7912

34. Rocchetta, H.L., Burrows, L.L., and Lam, J.S. 1999. Genetics of O-antigen biosynthesis in Pseudomonas aeruginosa. Microbiol. Mol. Biol. Rev. 63:523-553.

35. Comer, J.E., Marshall, M.A., Blanch, V.J., Deal, C.D., and Castric, P. 2002. Identification of the Psendomonas aeruginosa 1244 pilin glycosylation site. Infect. Immun. 70:2837-2845.

36. Schirm, M., et al. 2004. Structural and genetic characterization of glycosylation of type a flagellin in Pseudomonas aeruginosa. J. Bacteriol. 186:2523-2531.

37. Lewis, A.L., Hensler, M.E., Varki, A., and Nizet, V. 2006. The group B streptococcal sialic acid O-acetyltransferase is encoded by neuD, a conserved component of bacterial sialic acid biosynthetic gene clusters. J. Biol. Chem. 281:11186-11192.

38. Schoenhofen, I.C., et al. 2006. Functional characterization of dehydratase/aminotransferase pairs from Helicobacter and Campylobacter: enzymes distinguishing the pseudaminic acid and bacillosamine biosynthetic pathways. J. Biol. Chem. 281:723-732.

39. Vuong, C., et al. 2004. A crucial role for exopolysaccharide modification in bacterial biofilm formation, immune evasion, and virulence. J. Biol. Chem. 279:54881-54886.

40. Costerton, J.W. 2002. Anaerobic biofilm infections in cystic fibrosis. Mol. Cell. 10:699-700.

41. Singh, P.K., et al. 2000. Quorum-sensing signals indicate that cystic fibrosis lungs are infected with bacterial biofilms. Nature. 407:762-764.

42. Landry, R.M., An, D., Hupp, J.T., Singh, P.K., and Parsek, M.R. 2006. Mucin-Psendomonas aeruginosa interactions promote biofilm formation and antibiotic resistance. Mol. Microbiol. 59:142-151.

43. Walker, T.S., et al. 2005. Enhanced Pseudomonas aeruginosa biofilm development mediated by human neutrophils. Infect. Immun. 73:3693-3701.

44. Greiner, L.L., et al. 2004. Nontypeable Haemophilus 
influenzae strain 2019 produces a biofilm containing $\mathrm{N}$-acetylneuraminic acid that may mimic sialylated O-linked glycans. Infect. Immun. 72:4249-4260.

45. Donlan, R.M., et al. 2004. Model system for growing and quantifying Streptococcus pneumoniae biofilms in situ and in real time. Appl. Environ. Microbiol. 70:4980-4988.

46. Jurcisek, J., et al. 2005. Role of sialic acid and complex carbohydrate biosynthesis in biofilm formation by nontypeable Haemophilus influenzae in the chinchilla middle ear. Infect. Immun. 73:3210-3218.

47. Moncla, B.J., Braham, P., and Hillier, S.L. 1990. Sialidase (neuraminidase) activity among gramnegative anaerobic and capnophilic bacteria. J. Clin. Microbiol. 28:422-425.

48. Shakhnovich, E.A., King, S.J., and Weiser, J.N. 2002. Neuraminidase expressed by Streptococcus pneumoniae desialylates the lipopolysaccharide of Neisseria meningitidis and Haemophilus influenzae: a paradigm for interbacterial competition among pathogens of the human respiratory tract. Infect. Immun. 70:7161-7164.

49. Tong, H.H., Blue, L.E., James, M.A., and DeMaria,
T.F. 2000. Evaluation of the virulence of a Streptococcus pneumoniae neuraminidase-deficient mutant in nasopharyngeal colonization and development of otitis media in the chinchilla model. Infect. Immun. 68:921-924.

50. Probert, H.M., and Gibson, G.R. 2002. Bacterial biofilms in the human gastrointestinal tract. Curr. Issues Intest. Microbiol. 3:23-27.

51. Crennell, S.J., et al. 1996. The structures of Salmonella typhimurium LT2 neuraminidase and its complexes with three inhibitors at high resolution. J. Mol. Biol. 259:264-280.

52. Ada, G.L., French, E.L., and Lind, P.E. 1961. Purification and properties of neuraminidase from Vibrio cholerae. J. Gen. Microbiol. 24:409-425.

53. Moustafa, I., et al. 2004. Sialic acid recognition by Vibrio cholerae neuraminidase. J. Biol. Chem. 279:40819-40826.

54. Gallant, C.V., et al. 2005. Common beta-lactamases inhibit bacterial biofilm formation. Mol. Microbiol. 58:1012-1024.

55. Rothe, B., Roggentin, P., and Schauer, R. 1991. The sialidase gene from Clostridium septicum: cloning, sequencing, expression in Escherichia coli and identification of conserved sequences in sialidases and other proteins. Mol. Gen. Genet. 226:190-197.

56. Wolfgang, M.C., Lee, V.T., Gilmore, M.E., and Lory, S. 2003. Coordinate regulation of bacterial virulence genes by a novel adenylate cyclase-dependent signaling pathway. Dev. Cell. 4:253-263.

57. Ratner, A.J., et al. 2001. Cystic fibrosis pathogens activate $\mathrm{Ca}^{2+}$-dependent mitogen-activated protein kinase signaling pathways in airway epithelial cells. J. Biol. Chem. 276:19267-19275.

58. O'Toole, G.A., Gibbs, K.A., Hager, P.W., Phibbs, P.V., Jr., and Kolter, R. 2000. The global carbon metabolism regulator $\mathrm{Crc}$ is a component of a signal transduction pathway required for biofilm development by Psendomonas aeruginosa. J. Bacteriol. 182:425-431.

59. Davies, D.G., et al. 1998. The involvement of cellto-cell signals in the development of a bacterial biofilm. Science. 280:295-298.

60. Gomez, M.I., et al. 2004. Staphylococcus aureus protein A induces airway epithelial inflammatory responses by activating TNFR1. Nat. Med. 10:842-848. 